Check for updates

Cite this: RSC Adv., 2017, 7, 38019

\title{
The effect of organic additives on the intergranular conductivity of Al-doped $\mathrm{ZnO} \uparrow$
}

\author{
J. Gamon, (DD *ab D. Giaume, (DD ${ }^{a}$ G. Lefèvre, ${ }^{a}$ T. Le Mercier ${ }^{b}$ and P. Barboux ${ }^{a}$
}

The development of ink-based solutions as alternative routes to vacuum-based deposition techniques requires methods to improve their intergranular conductivity without thermal sintering. The effect of organic additives on the interparticle electron transfer between grains of an Al-doped ZnO powder was studied. The resistivity of the modified powders was measured as a function of compression to reach a reproducible state. The surface adsorption of selected molecules strongly enhances intergranular electron transfer, up to a factor of 20. This improvement has been linked to the decrease of the intergranular potential barrier height, associated with the energy distance between the Fermi level of the oxide and the LUMO of the molecules.

Received 12th March 2017

Accepted 21st July 2017

DOI: 10.1039/c7ra02975f

rsc.li/rsc-advances

in the literature ${ }^{4}$ (sol gel, ${ }^{16}$ chemical bath deposition, ${ }^{17}$ spray

\section{Introduction}

Deposition of colloidal inks have attracted much attention over the past decades as an alternative to vacuum-based thin films deposition technics. ${ }^{1-5}$ The method relies on the coating of an ink - the active material mixed in a solvent with additives to be subsequently removed - on a substrate using processes such as roll to roll, doctor blade, serigraphy, inkjet printing. ${ }^{6}$ The major advantage being the reduced investment cost and large scalability, this deposition route is also well suited for materials requiring soft chemical treatment, deposition onto plastic substrates or integration into hybrid organic/inorganic systems. However, a disadvantage of these solution techniques is that the resulting films are poorly densified and may contain residual organic impurities used during the ink formulation. This is the case of oleylamine, a very good particle stabilizer in most solvents that limits the conductivity in semiconductor films. ${ }^{3,5}$ Most of the time, an annealing treatment is required to densify the film and remove the insulating impurities..$^{7-9}$ However, this sintering step often induces cracks as well as carbon-based impurities at the grain boundaries, and is again not suited for materials with low degradation temperatures (the active material itself, the substrate or other sensitive films composing the device).

An example of this drawback is related to the case of transparent conducting oxides (TCOs) which are used as front contacts in photovoltaic devices or connectors in many display applications. Their conductivity is crucial for the efficiency of the device. Many solution-based processes have been described

${ }^{a}$ Chimie ParisTech, PSL Research University, CNRS, Institut de Recherche de Chimie Paris (IRCP), 75005 Paris, France. E-mail: jacinthe.gamon@chimie-paristech.fr

${ }^{b}$ Solvay, Research and Innovation Center of Paris (RIC Paris), 52 rue de la Haie-Coq, 93306 Aubervilliers, France

$\dagger$ Electronic supplementary information (ESI) available. See DOI: $10.1039 / \mathrm{c} 7 \mathrm{ra02975f}$ pyrolysis $^{18}$ ), for which the resistivity reaches down to $2 \times 10^{-3}$ $\Omega \mathrm{cm}$ for ITO thin films. ${ }^{19}$ However vacuum-based deposition routes (typically sputtering ${ }^{20}$ or pulsed laser deposition ${ }^{21}$ ) still offer the best performances with a resistivity still lower by one order of magnitude and reaching down to $10^{-4} \Omega \mathrm{cm}$ (sputtered $\mathrm{ITO}^{20,22}$ ). Therefore, solutions to obtain highly conducting liquid-based and low temperature processed thin films need to be further developed. For that purpose, a large effort must be put on improving the intergranular electrical transfer in these materials.

Most efforts focus on the ink formulation, using conducting ligands which stabilize the suspension prior to the deposition but also act as sintering agents after deposition. ${ }^{10,11}$ Ligand removal $^{12,13}$ or ligand exchange $\mathrm{e}^{14,15}$ with more conducting ones can also be performed.

Among TCO materials ZnO:Al has the additional advantages to be non-toxic and earth-abundant, which is one of the reason for its interest in the industry, especially in photovoltaics. Optimization of dye-sensitized solar cells already focused on the improvement of the electrical transfer between adsorbed molecules and this semiconductor. Previous works demonstrated that the grain-to-grain electronic transfer in a $\mathrm{ZnO}$ compact depends on the nature of the molecules sandwiched between the grains. ${ }^{10,32}$ The best results are bound to the position of the lowest unoccupied molecular orbitals (LUMO) of these molecules above the conduction band of the n-type semiconductor. ${ }^{23,24}$

When considering the case of an n-type semiconductor, such as $\mathrm{ZnO}: \mathrm{Al}$, the main charge carriers are electrons that diffuse through the conduction band. Their energy can therefore be estimated as the energy at the minimum of the conduction band, $E_{\mathrm{CB}}$. In polycrystalline semiconductor assemblies, the energy levels at the particles surface differ from the bulk, as the 
surface gathers carrier traps coming either from dangling bonds, from a change in chemical composition compared to the bulk, or from adsorbed surface species (such as water, oxygen...). ${ }^{25}$ Segregation of impurities at grain boundaries also occurs due to a size mismatch, or coulombic interactions. ${ }^{26-28}$ These defects trap electrons or holes and an energy band bending occurs on both sides of the grain boundary to establish electronic equilibrium. ${ }^{29-31}$ This phenomenon is at the origin of the so-called double Schottky barrier, the height of which depends on the intergranular domain. In areas where particles are not in contact, the intergranular domain may be considered as vacuum, and the barrier height is equal to the difference between the vacuum level and the conduction band level (Fig. 1a). ${ }^{32}$ When particles are in contact, the height of the potential barrier, $\Phi_{\mathrm{B}, \mathrm{GB}}$, depends on the excess charge of main carriers trapped at the interface (the grain boundary) (Fig. 1b), it can be determined for example by the energy position of the surface states. ${ }^{30}$ If an organic molecule is introduced in the intergranular domain (Fig. 1c), the barrier height becomes equal to the difference between the LUMO of the molecule and the electron energy since for an n-type semiconductor, charge carriers transfer through the LUMO. Respectively, in the case of p-type semiconductors, they would transfer through the HOMO of the molecules.

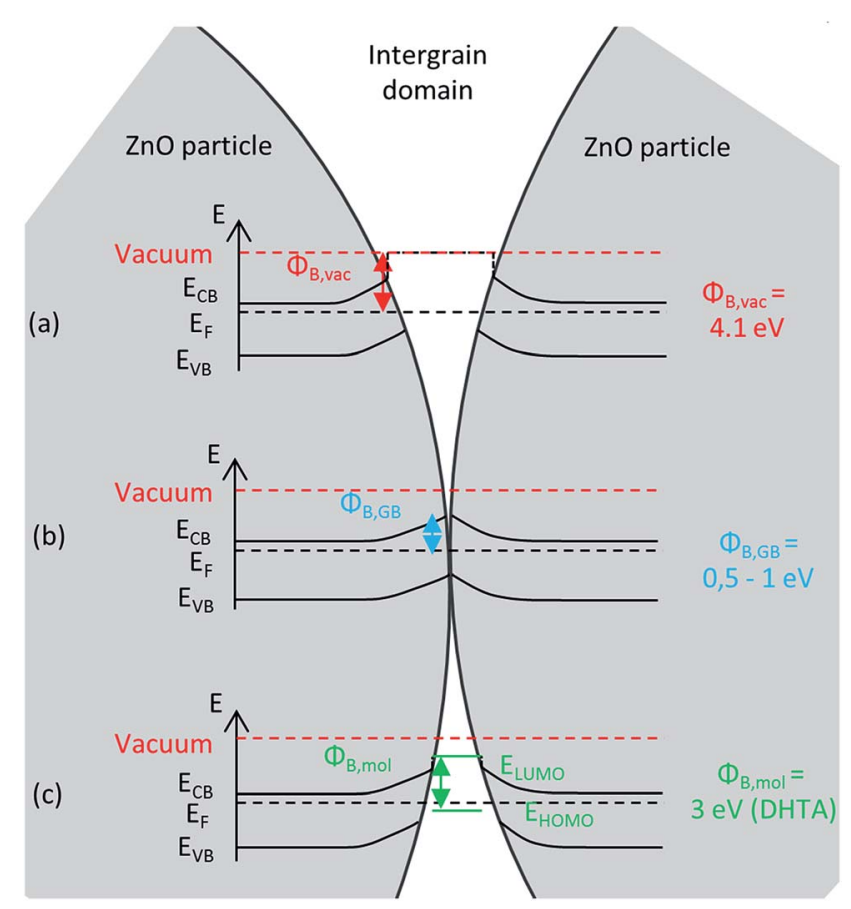

Fig. 1 Energy barrier at the interface between two particles of a ndoped semiconductor. All levels are taken with respect to the vacuum level (in red on the drawing). Three cases are considered: (a) the surfaces do not touch, the barrier height is $\Phi_{\mathrm{B}, \mathrm{vac}}$ ( (b) the particles touch, the barrier height is determined by the grain boundary properties $\Phi_{\mathrm{B}, \mathrm{GB}}$ and (c) an organic molecule links the semiconductor grains, the barrier height depends on the alignment of $E_{\mathrm{F}}$ and $E_{\mathrm{LUMO}}: \Phi_{\mathrm{B}, \mathrm{mol}}$. Average magnitudes of the different barrier height for $\mathrm{n}$ doped $\mathrm{ZnO}$ are indicated on the right. DHTA is taken as an example of molecule and stands for 2,5-dihydroxyterephthalic acid.
This energy barrier affects intergranular electronic transport. There are three ways for an electron in the conduction band to overcome the barrier and therefore contribute to the conductivity: (i) thermionic emission, if the thermal energy is higher than the barrier height, and (ii) tunneling across the barrier and (iii) transfer through surface states. Tunneling is described by quantum mechanics and the probability for the electron to cross the barrier, $T$, can be described by eqn (1): ${ }^{10,32}$

$$
T=\exp \left(-\frac{2}{\hbar} \sqrt{2 m \Phi_{\mathrm{B}}} L\right)
$$

To summarize, the intergranular electron transfer will be favored, when particles will be in close contact or when an organic molecule with a suitable LUMO will separate the two grains.

In this work, we discuss the grain-to-grain electronic transfer in a $\mathrm{ZnO}: \mathrm{Al}$ powder as a function of the nature of the molecules sandwiched between the grains. ${ }^{\mathbf{1 0 , 3 2}}$ We examined the influence of the LUMO energy levels of different organic additives on $\mathrm{ZnO}: \mathrm{Al}$ intergranular conductivity. Our goal is to establish a direct link between (i) the improvement of the intergranular electronic conductivity of a semiconductor and (ii) the energy position of an organic molecule adsorbed at its surface.

We chose to study the conductivity of a compressed powder at relatively low pressure (between 1 and $70 \mathrm{MPa}$ of uniaxial pressure) in order to obtain reproducible results. This is also an artificial manner to reproduce experimental conditions of wet deposited thin films where a high porosity is maintained and the contact pressure results from capillary effects.

\section{Experimental}

\section{A. DFT calculations}

The Frontier orbitals energy levels of the organic molecules were calculated thanks to ground state geometry optimization and frequency calculations using DFT method with the global hybrid functional PBE1PBE, using Gaussian basis sets 6-31G(d). The wave function type is unspecified and water is used as implicit solvent.

\section{B. Materials}

An aluminum-doped zinc oxide powder (3 wt $\%$ ) was purchased from Umicore (Zano ${ }^{\circledR} \mathrm{Al}-10$ Powder). It consists of nanosized particles (Fig. S0 $\dagger$ ). The surface area of the as-purchased powder is $30 \mathrm{~m}^{2} \mathrm{~g}^{-1}$. All experiments with organic additives were performed with a batch of powder produced by heating the commercial powder in a tubular furnace under an $\mathrm{H}_{2} / \mathrm{Ar}(4 \%)$ atmosphere at $900{ }^{\circ} \mathrm{C}$ for 1 hour with heating and cooling ramps of $5{ }^{\circ} \mathrm{C} \min ^{-1}$. After annealing, the surface area of the powder decreased down to $2 \mathrm{~m}^{2} \mathrm{~g}^{-1}$. A sintered pellet was obtained by pressing this annealed $\mathrm{ZnO}: \mathrm{Al}$ powder under an uniaxial press at $100 \mathrm{MPa}$, after which the same thermal treatment under $\mathrm{H}_{2}$ was performed a second time. The compactness of the pellet before sintering is $63 \%$, and $70 \%$ after sintering. 
Succinic acid (SA), 99\%; terephtalic acid (TA), 98\%; 2-nitroterephthalic acid (NTA), 99\%; 3,5-dinitrobenzoic acid (DNBA), 99\%; 2,5-diaminoterephthalic acid (DATA), 95\%, 2,5-dihydroxyterephthalic acid (DHTA), 98\% and poly(thiophene-3-[2-(2methoxyethoxy)ethoxy]-2,5-diyl), sulfonated solution (PTS) 2\% in 1,2-propanediol : isopropanol : water, $3: 2: 1$, were all purchased from Sigma Aldrich.

\section{Techniques and equipment}

A Flash 2000 analyzer (ThermoFisherScientific) was used for the quantitative determination of carbon in the Al-doped $\mathrm{ZnO}$ powder grafted with terephtalic acid derivatives and succinic acid. Measurements were performed and repeated 3 times on $10 \mathrm{mg}$ of sample. Quantitative mass determination of the grafted polymer in the PTS-functionalized Al-doped $\mathrm{ZnO}$ powder was performed by thermogravimetric analysis under oxygen, thanks to a Netzsch STA 449F3 TGA-TDA apparatus.

Resistivity measurements as a function of temperature were obtained with the four probes method. Contacts were made with a silver paste (purchased from SPI supplies). This was used to compare the effect of $\mathrm{H}_{2}$ annealing and sintering.

The specific surface area of the powders were measured thanks to the method Brunauer, Emmett and Teller (BET) with a Belsorp apparatus.

\section{Functionalization procedure}

The organic additives studies were performed on the $\mathrm{H}_{2} / \mathrm{Ar}$ annealed ZnO:Al powder, which presented the most reproducible conductivity.

Carboxylate additives. Carboxylate additives (SA, TA, DNBA, DHTA, DATA, NTA, see the full organic names in Fig. 4(a)). 200 $\mathrm{mL}$ of deprotonated acid solution at $10^{-3} \mathrm{M}$ were obtained by dissolving the appropriate weight in water thanks to successive additions of $1 \mathrm{M} \mathrm{NaOH}$ solution in order to reach a pH of 7.5. This $\mathrm{pH}$ is essential to prevent the dissolution of $\mathrm{ZnO}: \mathrm{Al}$ in the solution and provides a good grafting of the carboxylate molecules as checked with IR spectroscopy. $2 \mathrm{~g}$ of annealed $\mathrm{ZnO}: \mathrm{Al}$ was dispersed thanks to vigorous stirring in each of these carboxylate solutions and maintained overnight under agitation. The suspensions were then filtered through a Büchner funnel without further washing. The resulting powders were dried overnight at $70{ }^{\circ} \mathrm{C}$, and ground in an agate mortar.

PTS additives. A commercial solution of poly(thiophene-3-[2(2-methoxyethoxy)ethoxy]-2,5-diyl), sulfonated solution, at 2 wt\% in 1,2-propanediol : isopropanol : water, $3: 2: 1$ was used for the functionalization of $\mathrm{ZnO}: \mathrm{Al}$ with the polythiophene. 1 $\mathrm{mL}$ of this solution was diluted in $200 \mathrm{~mL}$ of water, which corresponds to a polymer concentration of approximately $10^{-4}$ M. $2 \mathrm{~g}$ of annealed $\mathrm{ZnO}: \mathrm{Al}$ powder was dispersed in the solution thanks to vigorous stirring, yielding a weight ratio PTS/ $\mathrm{ZnO}$ : Al of $1 \mathrm{wt} \%$. The suspension was kept under agitation overnight and filtered through a Büchner funnel without further washing. The powder was then dried at $150{ }^{\circ} \mathrm{C}$ in order to thoroughly remove the organic solvents present in the commercial solution of PTS.
Nature of adsorption and amount of molecules adsorbed. Infrared spectroscopy measurements enabled to validate that an adsorption occurs between the molecules and the semiconductor ( $c f$. ESI, $\dagger$ part 2). The nature of the adsorption of the molecule at the surface of the semiconductor can be considered either as an electrostatic interaction, or a covalent grafting. Our goal in this paper is not to distinguish both types of adsorption, as we believe they both play a role in the modification of the intergranular conductivity. We argumented in the last section (D. Discussion), why models for explaining electron transfer can be considered the same for both types of interaction. Therefore, the effect of adsorbed additives in general will be considered and the powder is not washed after immersion to avoid molecule removal.

In order to determine the quantity of molecules present at the surface of the grafted $\mathrm{ZnO}$ :Al particles, carbon and nitrogen were quantified thanks to CHNS analysis for carboxylate molecules, whereas TGA was performed for the PTS functionalization. Indeed, the amount of carbon is not exactly known in the polymer, and CHNS analysis would not enable to determine the exact amount of molecules adsorbed. This analysis reveals a weight percentage between $0.15 \%$ and $0.35 \%$ for the carboxylate molecules, and $0.81 \%$ for the sulfonated polythiophene.

Taking into account the specific surface of the powder, $2 \mathrm{~m}^{2}$ $\mathrm{g}^{-1}$, this corresponds to 2.5 to 3.8 molecules adsorbed per $\mathrm{nm}^{2}$. This value is high and probably indicates some residual ungrafted molecules. The effect of the amount of adsorbed molecules was therefore considered. For this experiment, the powder was immersed in aqueous solutions of different concentration of molecules, then only dried (and not filtered) to control the exact amount of molecule intimately dispersed with the $\mathrm{ZnO}$ :Al powder.

\section{E. Experimental set-up for resistivity measurements under pressure}

The set-up used for the resistivity measurements is similar to that of Celzard et $a .^{33}$ and has already been presented by our group. ${ }^{34}$ A 13 mm-diameter stainless steel cylindrical swagelock cell equipped with two plungers and internally covered by a Mylar sheet to provide electrical insulation was filled with the appropriate mass of powder. The powder surface was first flattened thanks to taps on the bench, before delicately placing the whole cell between two compression moving platens of a numerically monitored Instron 5966 testing system (Fig. 2a). The displacement rate of the upper compression plate was imposed, and the resultant force applied on the cell was recorded. The full profile of compression cycle is described in Fig. $2 \mathrm{~b}$.

The powder thickness was directly related to the platens displacement, setting first the zero displacement on the empty cell pressed under $73 \mathrm{MPa}$ (maximum pressure), and for each pressure, systematically subtracting afterwards the displacement due to the cell deformation from the displacement recorded with powder.

The two plungers were connected to a Keithley 2400 ohmmeter, which recorded the two probe resistance $R$, between the plungers during the compression cycle. Because two probe resistance measurements may include junction resistance 
(a)

(b)

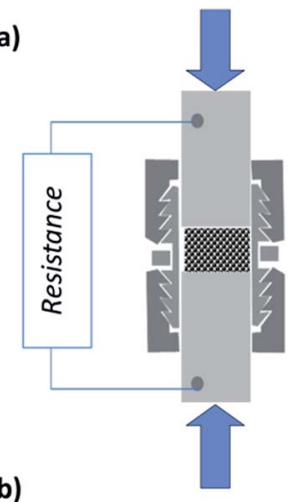

$9500 \mathrm{~N}(73 \mathrm{MPa}), 1 \mathrm{~min}$

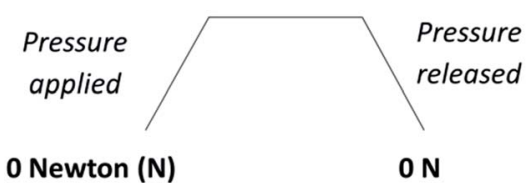

Fig. 2 (a) Set-up for resistivity measurements of the $\mathrm{ZnO}$ :Al powder under pressure. (b) Profile of the compression cycle.

contribution, a preliminary calibration was performed with different amounts of powder to validate that the resistance was linearly linked to the calculated thickness. The extrapolation of the resistance at zero mass accounts for the junction resistance and stays below $0.4 \Omega$. This negligible junction resistance was not taken into account in our results, and only comparisons between samples were made.

The resistivity was obtained by the formula:

$$
\rho=R \times \frac{S}{e}
$$

where $S$ is the plunger surface $\left(1.32 \mathrm{~cm}^{2}\right)$ and $e$ the calculated thickness. The relative density of the samples $d_{\mathrm{R}}$ was determined by eqn (2):

$$
d_{\mathrm{R}}=\frac{m /(S \times e)}{d_{\mathrm{b}}}
$$

where $m$ is the mass of the powder $(0.7 \mathrm{~g})$, and $d_{\mathrm{b}}$ the bulk density of zinc oxide $\left(5.6 \mathrm{~g} \mathrm{~cm}^{-3}\right)$.

\section{Results and discussion}

\section{A. Improving bulk electrical conductivity}

The resistivity of the as-purchased $\mathrm{Al}$-doped $\mathrm{ZnO}$ was measured with the four probe method on a pellet pressed at $100 \mathrm{MPa}$. Its compactness is $63 \%$, and its resistivity is $5 \times 10^{4} \Omega \mathrm{cm}(300 \mathrm{~K})$ with a thermally activated behavior as for a semiconductor $\left(E_{\mathrm{a}}=\right.$ $0.24 \mathrm{eV}$ ) (Fig. 3a). This result was obtained on a pellet previously dried at $100{ }^{\circ} \mathrm{C}$ for $12 \mathrm{~h}$ in an oven. We observed a strong irreproducibility during this measurement and realized that the water vapor pressure (relative humidity) had a strong effect on the conductivity. This effect is attributed to surface proton diffusion. The conducting properties of the semiconductor zinc oxide are known to be very sensitive to adsorbed species such as oxygen, carbon monoxide. To avoid non-reproducible measurements due to surface effects, ${ }^{35,36}$ doping of zinc oxide is required so that its intrinsic electronic conductivity becomes higher than proton surface conductivity and remains weakly altered by surface states.

Thus, the powder was annealed in a tube furnace under $\mathrm{H}_{2} /$ $\mathrm{Ar}$ at $900{ }^{\circ} \mathrm{C}$ before pelletizing. This considerably decreased the resistivity of the pellet down to $1 \mathrm{k} \Omega \mathrm{cm}(300 \mathrm{~K})$ with an activation energy of $0.1 \mathrm{eV}$ (Fig. 3b), while slightly increasing its compactness to $68 \%$. An annealing treatment under a reducing atmosphere $\left(5 \% \mathrm{H}_{2} / \mathrm{Ar}\right)$ at $900{ }^{\circ} \mathrm{C}$ is well known to be very beneficial as suggested in the literature. ${ }^{37-39}$

This increased conductivity after thermal treatment is probably due to a better aluminum insertion into the zinc oxide structure. Indeed, the annealing should shifts the equilibria of the following reaction (eqn (3)) towards the right, therefore increasing the doping by aluminum and the amount of charge carriers:

$$
\mathrm{Al}_{2} \mathrm{O}_{3} \stackrel{\mathrm{ZnO}}{\longrightarrow} 2 \mathrm{Al}_{\mathrm{Zn}}^{\cdot}+2 \mathrm{O}_{\mathrm{O}}^{\times}+\frac{1}{2} \mathrm{O}_{2}+2 \mathrm{e}^{\prime}
$$

Moreover, the desorption of negatively charged oxygen species at the grain boundaries during annealing may result in a higher carrier mobility and also contributes to the increase in conductivity. ${ }^{38,39}$

This annealing procedure is important to obtain a reproducible intrinsic conductivity in the grains.

A similar pellet was also sintered (i.e. as a ceramic) at $900{ }^{\circ} \mathrm{C}$ - for $12 \mathrm{~h}$ under $\mathrm{H}_{2} / \mathrm{Ar}$. The compactness of the resulting ceramic was $70 \%$. But its resistivity falls down to $4 \times 10^{-1} \Omega \mathrm{cm}$ at $300 \mathrm{~K}$ and is nearly independent of the temperature, characteristic of a metallic behavior (Fig. 3c). This demonstrates that

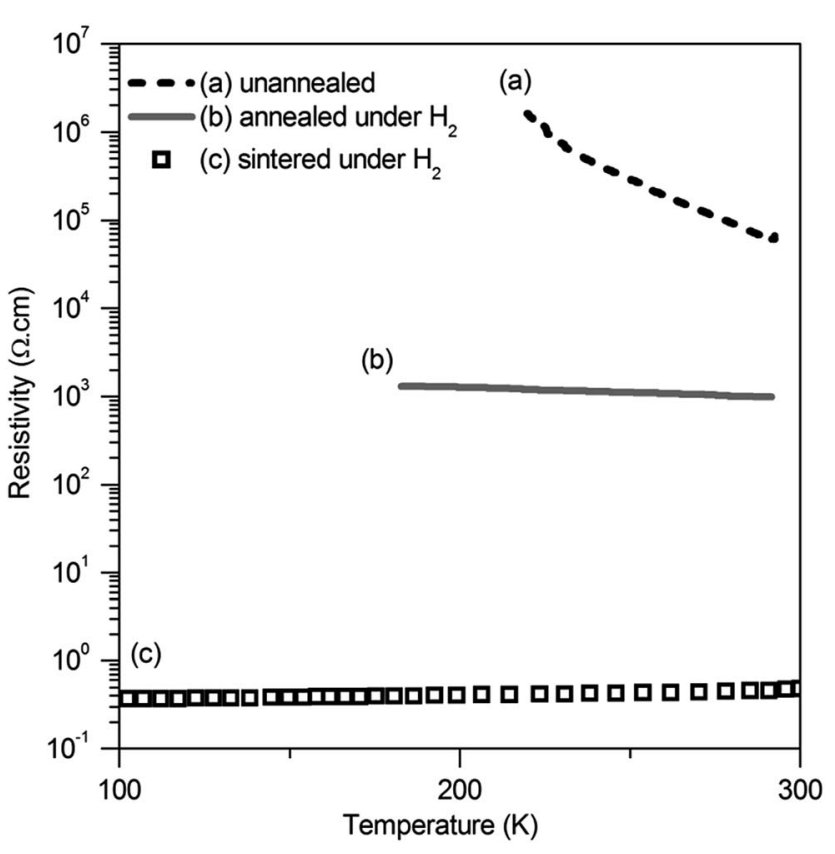

Fig. 3 Resistivity of pressed pellets as a function of the temperature for pressed $\mathrm{ZnO}$ :Al powders, (a) unannealed powder and (b) annealed under $\mathrm{H}_{2}$. (c) Resistivity of a pellet sintered at $900{ }^{\circ} \mathrm{C}$ under $\mathrm{H}_{2}$. 
by improving intergranular contacts in the pellet, the resistivity can decrease at least by 3 orders of magnitude lower than the simply pressed powder. This high conductivity value may be considered as an upper limit for the intergranular conductivity of the grains and, as a matter of fact, the goal of the following studies with organic additives would be to reach a similar value by improving intergranular electronic transfers, with room temperature particle surface functionalization instead of high temperature sintering.

\section{B. Choice of organic molecule and energy diagram}

A range of small molecules, presented in Fig. 4a, was selected to study the influence of functionalization on $\mathrm{ZnO}$ :Al intergranular conductivity, and more specifically, the influence of an anchoring group, or a conjugated pi system on the molecules. Terephthalic acid and its analogues revealed to be appropriate for this study due to their carboxylate function, known to be a good anchoring group at the surface of zinc oxide, ${ }^{24}$ as well as for their conjugated pi systems favoring electronic transfer. The substituting groups were selected in order to obtain different LUMO levels. Succinic acid, an aliphatic molecule possessing the same carboxylate function, was added to the study. Its LUMO level is higher. A substituted polythiophene (PTS) was also selected as it is known to be a good electronic mediator, as well as possessing a low LUMO level. ${ }^{\mathbf{4 0 , 4 1}}$ Energy levels of the isolated molecules were calculated thanks to DFT calculations, and positioned relatively to the conduction and valence band levels of $\mathrm{ZnO}$ (Fig. 4b).

\section{Effect of organic molecules on the intergranular conductivity}

The resistivity of the $\mathrm{ZnO}$ :Al powder functionalized with the different molecules has been studied at room temperature as a function of the applied pressure. The relative density of the powder under compression is plotted on Fig. S4, $\dagger$ and shows that the powder compaction is not affected by molecule grafting.

The change of the resistivity versus the applied pressure is presented on a log-log plot on Fig. 5. For the sake of clarity, it is only shown for a selection of most representative molecules. Below $10^{6} \mathrm{~Pa}$, the powders are only weakly densified and remain very loose, so their measurements are very irreproducible. Under higher pressure, a strong decrease of the resistivity is observed, during which elastic and plastic deformations take place. The elastic phenomena can be estimated by the increase of resistivity upon the pressure release. The plastic contribution is indicated by the resulting hard pellet, which only returns to powder if ground in a mortar. If the pressure is increased typically to $10^{9} \mathrm{~Pa}$ - a flat stage is obtained, corresponding to the end of the deformation. ${ }^{42}$

In Fig. 5, the resistivity and the pressure are represented in a logarithmic scale. A linear plot is observed for the ungrafted material, which is typically obtained for powders under compression. This indicates that the resistivity follows a powertype law: $\rho=q \times P^{\gamma}$, where $P$ is the pressure in $\mathrm{Pa}, q$ is a constant which contains contributions from the elastic modulus of the grain core and surface, the conductivity, the (a)

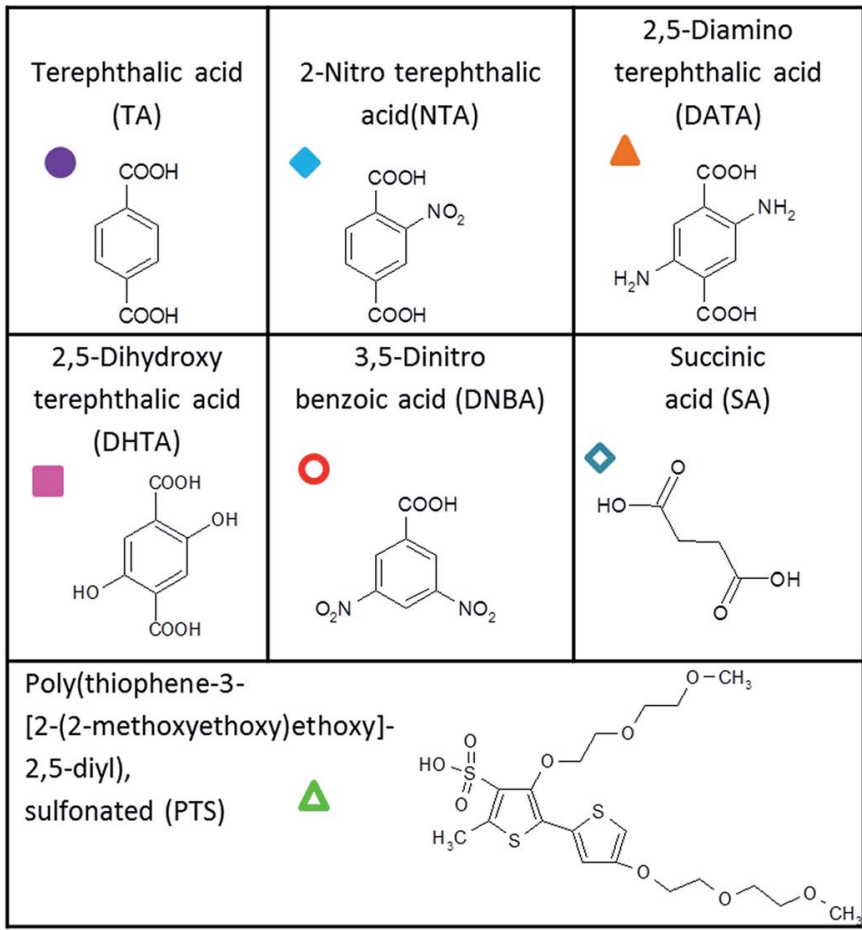

(b)

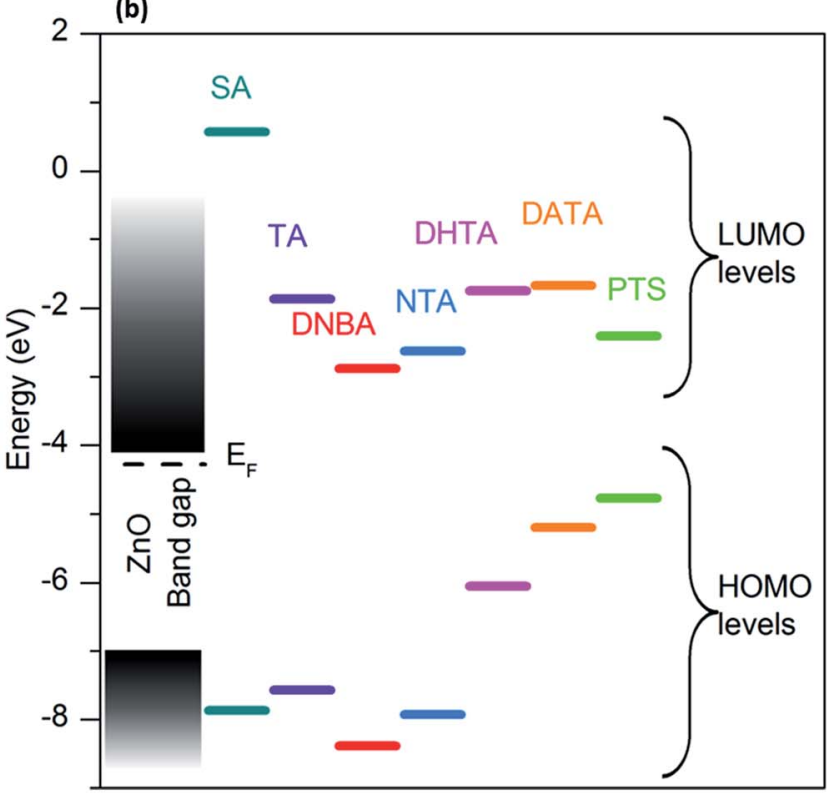

Fig. 4 (a) List of molecules anchored onto ZnO:Al surface and their names in the IUPAC system; (b) position of calculated HOMO and LUMO levels of anchoring molecules, and of the conduction and valence bands of $\mathrm{ZnO}$ compared to the vacuum level. 


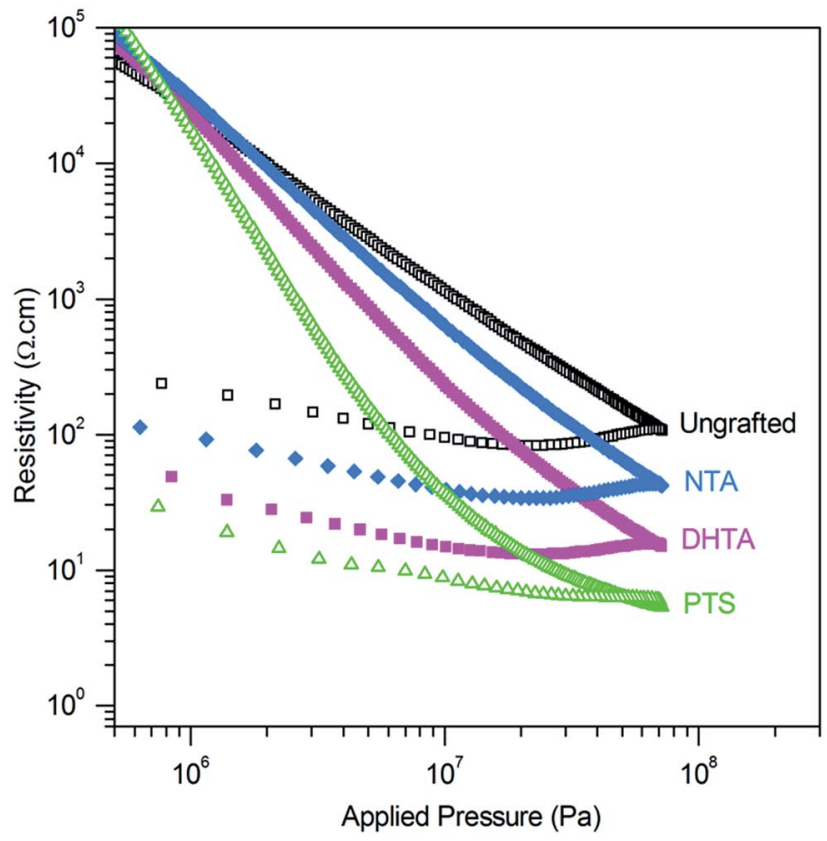

Fig. 5 Resistivity in function of the applied pressure on ungrafted and grafted $\mathrm{ZnO}$ :Al powder $\left(\mathrm{H}_{2} / \mathrm{Ar}\right.$ annealed powder) (anchoring molecules in legend).

grain diameter and the surface layer thickness. $\gamma$ is a dimensionless exponent, the value of which reflects the surface layer properties. For an ideal powder of spherical particles with no surface layer, the Hertz model, describing the surface contact area between particles as a function of the applied pressure, predicts that in the elastic regime $\gamma=-2 / 3 .^{42,43}$

The values of $\gamma$ obtained on our experimental curves is 1.2 for ungrafted ZnO:Al. The deviation from Hertz law for the grafted powders indicates the non-uniformity of the surface layer as well as the contribution of plastic deformations. ${ }^{43}$ All the resistivity measurements show an excellent reproducibility above $2 \times 10^{6} \mathrm{~Pa}$. All the molecules used in this study present a positive effect on the conductivity, except for the nonconjugated succinic acid, SA, which does not show any effect at all (the resistivity versus applied pressure exactly superposes with the ungrafted material in Fig. 6) Thus, a conjugated pisystem is necessary to improve the intergranular conductivity. The resistivity of the grafted powders becomes lower than the ungrafted material beyond 1 or $10 \mathrm{MPa}$, depending on the organic species. DATA and DHTA for instance decrease the resistivity by a factor of 5 . The sulfonated polythiophene polymer (PTS) has the largest effect: the resistivity drops dramatically and becomes 20 times lower (at $73 \mathrm{MPa}$ ) than for the ungrafted powder.

The conductivity increase is independent on the amount of molecules added to the surface. This was confirmed by additional experiments performed on powders immersed in aqueous solutions with different concentrations of molecules, then only dried (and not filtered) to control the exact amount of molecule intimately dispersed with the ZnO:Al powder. The PTS-grafted ZnO:Al had to be dried at higher temperature $\left(150{ }^{\circ} \mathrm{C}\right)$ to remove solvent residues. As the drying temperature

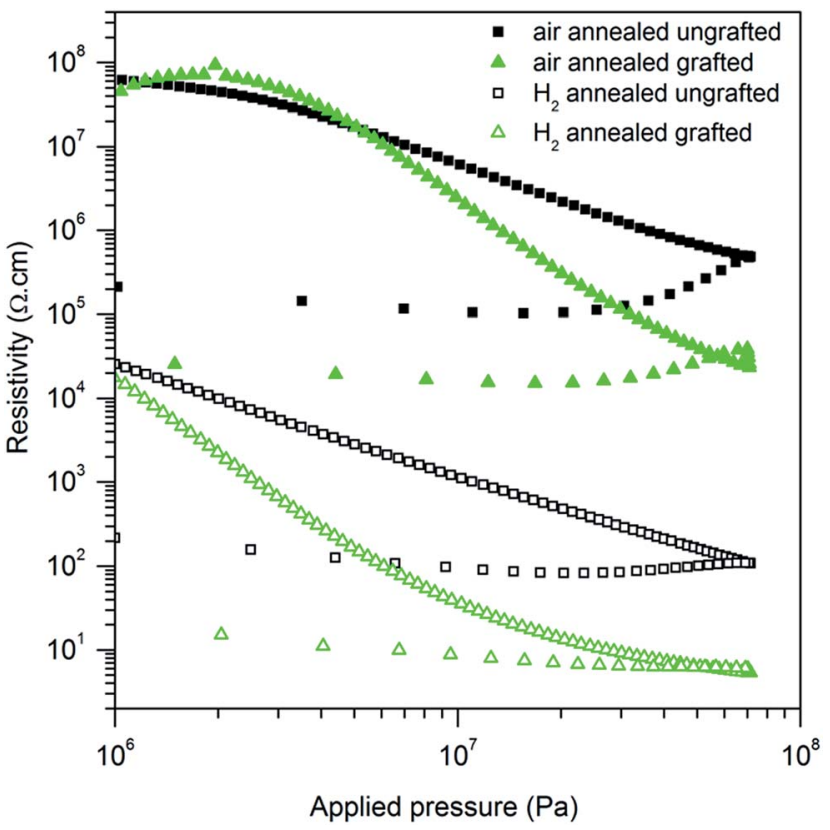

Fig. 6 Resistivity versus applied pressure of ungrafted (black squares) and PTS-grafted (green triangles) ZnO:Al powder annealed at $900{ }^{\circ} \mathrm{C}$ under air (full symbols), or under $\mathrm{H}_{2} / \mathrm{Ar}$ (empty symbols).

could be responsible for the conductivity enhancement, through the creation of carbon species for instance, the same drying process was performed on the ungrafted and NTAgrafted powders. In both cases, the conductivity of the powder dried at $150^{\circ} \mathrm{C}$ was lower than that of the powder dried at $70^{\circ} \mathrm{C}$, invalidating the role of temperature drying on the high conductivity of the PTS-grafted sample.

Moreover, this polymer is known to be a good conductor ${ }^{4-46}$ and is used as a p-type semiconductor in photovoltaic devices. ${ }^{47-49}$ Our goal here is to enable grain to grain electron transfer, but avoid polymer percolation, so that the bulk electrical properties of the material are still those of ZnO. In order to verify that no percolation of the polymer alone causes the electron transport, the resistivity of a poorly conducting $\mathrm{ZnO}: \mathrm{Al}$ powder (i.e. annealed under air in order to obtain the same specific surface as the powder annealed under $\mathrm{H}_{2}$ ) was measured before and after PTS-functionalization (Fig. 6).

The resistivity of the air-annealed powder is 3 orders of magnitude higher than that of the hydrogen-annealed powder, as discussed in part A. The PTS addition enables to increase the overall conductivity by one order of magnitude as compared to the raw powder. For the air-annealed and $\mathrm{H}_{2}$-annealed samples as well. If PTS percolation was responsible for the conductivity enhancement, the resistivity value of both functionalized samples would be equal and as low. As this is not the case, one can conclude that the resistivity enhancement is only due to improved intergranular contacts.

In Fig. 7, the resistivity observed at $70 \mathrm{MPa}$ has been plotted as a function of the difference between the energy levels of the LUMO and the HOMO of the grafted molecules. It is difficult to compare the results obtained for the functionalization with the carboxylate molecules with those obtained for the PTS 
functionalization as the chain length as well as the anchoring group is different. However, the positive effect of surface functionalization on the conductivity can be linked to the molecule HOMO-LUMO gap: $\Delta_{\mathrm{H}-\mathrm{L}}=E_{\mathrm{LUMO}}-E_{\mathrm{Hомо}}$. The smaller $\Delta_{\mathrm{H}-\mathrm{L}}$, the more conducting the compressed powder. This dependence will be explained in terms of energy level alignment in the next section.

\section{Discussion on energy level alignment and electron transfer}

The energy level alignment at the interface between a semiconductor or a metal and an organic molecule is difficult to estimate and has been a controversial issue. ${ }^{50,51}$ With the advances in the field of hybrid electronic devices such as sensors or DSSC, a growing interest towards this understanding has recently showed up to control charge transfer and performances.

First, before considering aligning the energy levels of the molecules with those of the solid, we must assess how the conduction and valence bands of the solid as well as the vacuum level are affected at the surface. Indeed, for a metal or a semiconductor, the potential well imposed by the work function is not infinitely deep. Therefore, there is a slight probability to find electrons outside this well: electrons are said to be leaking out of the surface. ${ }^{52,53}$ This results in the creation of a surface dipole, noted $\Delta_{\mathrm{s}}$, which means a change of the work function, $W_{\mathrm{s}}$, at the surface compared to that of the bulk, $W$ (Fig. 8a). Surface effects in n-type semiconductor tend to increase this dipole, because of acceptor states located at the surface which trap electrons.

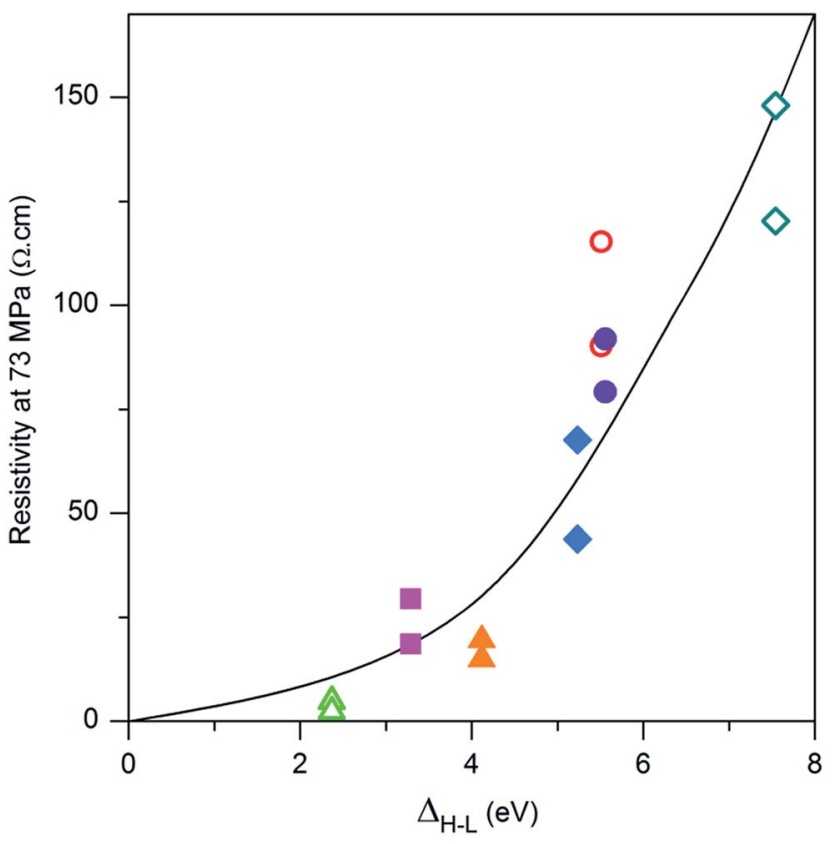

Fig. 7 Resistivity of grafted $\mathrm{ZnO}$ :Al powder $\left(\mathrm{H}_{2} / \mathrm{Ar}\right.$ annealed powder) under $73 \mathrm{MPa}$ in function of the energy difference $\Delta_{\mathrm{H}-\mathrm{L}}$. The color and sign represent the value (repeated twice) for a specific molecule (correspondences are given in Fig. 4).
When a molecule approaches the surface of a solid, electronic distribution rearrangement occurs upon interaction (charge transfer, mirror force, interface states, surface rearrangement...53), which contributes to a new change in the work function. Its amplitude strongly depends on the type of interaction electrostatic or chemical bonding for instance..$^{50,52-55}$ Many different effects have been described, mostly for metal/ organic interfaces, but these can be ascribed to semiconductors as well.

Recent studies aiming at developing a universal law for the alignment of energy levels between metals (or metal oxides) on one side and organic molecules converge towards the distinction of two regimes. ${ }^{56-58}$ First, for low interacting interfaces, no charge exchange occurs, and a common vacuum level is set between the molecule and the solid. This regime is said to follow the Schottky-Mott rule, which states that the energy levels are those of the solid and of the molecule taken separately, within a constant, noted $\Delta^{*}$, which depends on the physisorption mechanism, which itself introduces a dipole of amplitude $\Delta^{*}$ at the interface.

A main contribution to this dipole is called the push-back effect. It stipulates that the electronic cloud leaking out of the solid's surface is pushed back inside upon adsorption, and therefore the surface dipole and the work function are reduced. ${ }^{52}$ As a consequence, the energy levels of the molecules $\left(E_{\mathrm{HOMO}}^{*}\right.$ and $\left.E_{\mathrm{LUMO}}^{*}\right)$ are lowered with respect to the Fermi level, $E_{\mathrm{F}}$, of the solid.

This push-back dipole is always negative (Fig. 8b), and therefore lowers the surface work function $W_{\mathrm{s}}^{*}{ }^{*}{ }^{2,56}$ The amplitude of $\Delta^{*}$ has been proved to be similar for many different low interacting interfaces. ${ }^{56,57}$

The second regime occurs when a charge is transferred through the interface. According to Greiner et al. this charge transfer may take place when the ionization energy (or the electronic affinity) is smaller (or bigger) than the solid's work function..$^{56}$ In this case, the energy levels of the molecule are shifted regarding those of the solid: the HOMO (or LUMO) becomes pinned to the solid's Fermi level (not directly at the Fermi level, but separated by an energy $\varepsilon$, explained by Braun et al. by the electron polaronic binding energy ${ }^{57}$ ). The origin of this phenomenon is attributed by the authors to an electron transfer from the HOMO of the molecule to the solid (or from the solid to the LUMO), because the ionized molecules are thermodynamically more stable than their neutral form. This induces a new change in the surface dipole and a new surface work function, noted $\Delta_{\mathrm{t}}$ and $W_{\mathrm{s}}^{\mathrm{i}}$ respectively. Fig. 8c depicts this case. For any molecule such that EI $<W_{\mathrm{s}}$, the HOMO pinning regime occurs, the amplitude of $\Delta_{\mathrm{t}}$ is strongly dependent on the molecule, on the contrary to $\Delta^{*}$ established in the first regime.

In our system, before adsorption, the zinc oxide surface benefits from an upwards band bending. This situation is typical in n-type semiconductor, where electrons, coming from the conduction band, transfer to acceptor states at the surface (originating from dangling bonds, variation in chemical composition....). ${ }^{26-28,31}$ This leads to the enhancement of the work function at the interface as shown on Fig. 8a. As molecules draw near the surface, if the work function at the surface 


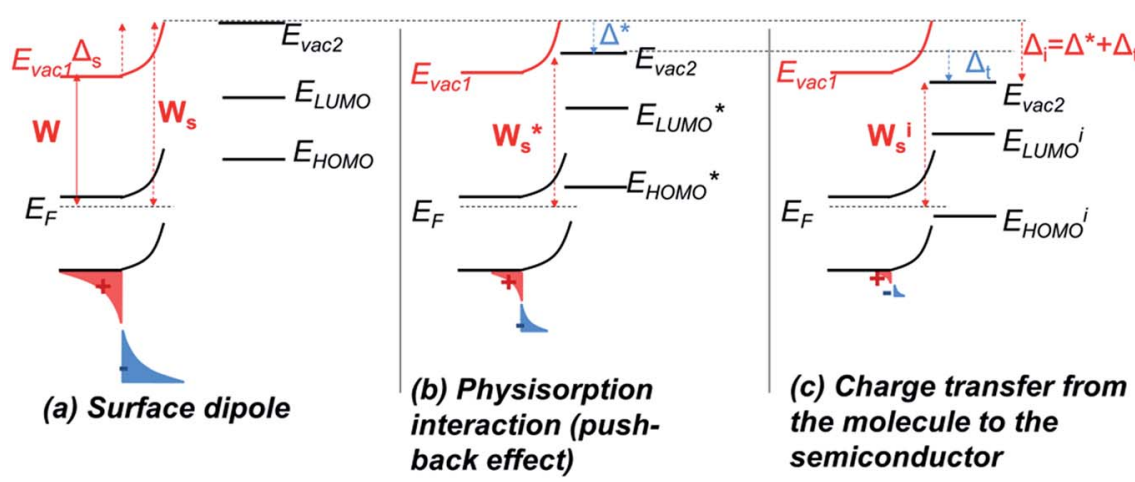

Fig. 8 Drawing of the energy levels at the surface of an $n$-type semiconductor before adsorption (a); when a physisorption interaction (such as the push-back effect) occurs between a molecule and the surface (b), leading to the creation of a dipole $\Delta^{*}$; when a charge transfer occurs from the molecule to the semiconductor leading to reduction of the overall surface dipole (addition of a negative contribution $\Delta_{t}$ ) (c).

becomes bigger than the ionization energy of the molecule, a charge transfer is favorable from the molecule to the solid, which itself results in the downward shift of the energy levels (negative $\Delta_{\mathrm{t}}$ ) down to the pinning of the HOMO to the Fermi level. This situation is the one described in Fig. 8.

Considering this hypothesis, the energy levels of the molecules have been repositioned assuming this hypothesis of Fermi level pinning (Fig. S5†). In this case, the energy barrier height, $\Phi_{\mathrm{B}, \mathrm{mol}}$, for electron transfer can be directly linked to $\Delta_{\mathrm{H}-\mathrm{L}}$. This conclusion would explain why the lower $\Delta_{\mathrm{H}-\mathrm{L}}$ induces a low potential barrier at the grain boundary through the introduction of a vacant energy level when the LUMO of the molecule is closest to the conduction band of the semiconductor. Concerning PTS functionalization, due to its long chain length (around 10 units), it is likely that a combined effect of the low potential barrier height as well as a higher amount of intergranular volume filled with the organic species takes place, enhancing even more the powder conductivity.

\section{Conclusion}

We have studied the influence of organic additives on the interparticle conductivity of ZnO:Al powders. The resistivity evolution of the powder under compression reveals that the surface adsorption of molecules strongly enhances intergranular electron transfer under pressures as low as $1 \mathrm{MPa}$. The effect is dominated by the position of the LUMO of the molecule as compared to the energy levels of the $\mathrm{ZnO}$ material. The most efficient organic additive, a sulfonated polythiophene, could even decrease the resistivity by a factor 20 at $73 \mathrm{MPa}$. This improvement is linked to a decrease of the intergranular potential barrier height, as well as to an increased contact area between particles. Further work should address the electron distribution and the electron transfer between the molecules and the solid surface. This study opens the way for a better conductivity control of thin films deposited by liquid-based processes, in which particles are not sintered and often show high intergranular resistivity. Functionalization using simple conjugated organics could therefore be an alternative to high temperature annealing steps, non-feasible for deposition on polymeric flexible substrates or for sensitive materials decomposing under high temperatures.

\section{Acknowledgements}

We thank Dr T. Le Bahers (Laboratoire de Chimie, ENS de Lyon, France) for performing DFT calculations and for sharing his knowledge about electron transfer in organic molecules, The Structural Metallurgy team at the Institut de Recherche de Chimie de Paris (IRCP, Chimie ParisTech, PSL Research University, France) for giving us access and time to their Ingstrom Press used for the conductivity measurements, Dr F. Decampo (Solvay, Bollate Research Center, Italy) and Dr J. Wilson (Solvay, Research and Innovation Center of Paris, France) for giving their advice and expertise on choosing and anchoring polythiophenes onto zinc oxide. We also thank $\mathrm{T}$. Barbier, S. Goyard, S. Croyeau and W. Win (Solvay, Research and Innovation Center of Paris, France) for performing ICP and CHNS analysis.

\section{Notes and references}

1 H. W. Choi, T. Zhou, M. Singh and G. E. Jabbour, Nanoscale, 2015, 7, 3338-3355.

2 M. M. Ling and Z. Bao, Chem. Mater., 2004, 16, 4824-4840.

3 S. E. Habas, H. A. S. Platt, M. F. A. M. van Hest and D. S. Ginley, Chem. Rev., 2010, 110, 6571-6594.

4 R. M. Pasquarelli, D. S. Ginley and R. O'Hayre, Chem. Soc. Rev., 2011, 40, 5406.

5 D. V. Talapin, J.-S. Lee, M. V. Kovalenko and E. V. Shevchenko, Chem. Rev., 2010, 110, 389-458.

6 J. Alex, der Vater des Computers, ed. K. Zuse, Parzeller, Fulda, 2000.

7 M. N. Kamalasanan and S. Chandra, Thin Solid Films, 1996, 288, 112-115.

8 M. Law, J. M. Luther, Q. Song, B. K. Hughes, C. L. Perkins and A. J. Nozik, J. Am. Chem. Soc., 2008, 130, 5974-5985.

9 J. W. Cho, A. Ismail, S. J. Park, W. Kim, S. Yoon and B. K. Min, ACS Appl. Mater. Interfaces, 2013, 5, 4162-4165. 
10 M. V. Kovalenko, M. Scheele and D. V. Talapin, Science, 2009, 324, 1417-1420.

11 C. Murray, E. Shevchenko and D. Talapin, International Business Machines Corporation, Columbia University, US Pat., US20070057255 A1, 2007.

12 M. V. Jarosz, V. J. Porter, B. R. Fisher, M. A. Kastner and M. G. Bawendi, Phys. Rev. B: Condens. Matter Mater. Phys., 2004, 70, 195327.

13 M. Grouchko, A. Kamyshny, C. F. Mihailescu, D. F. Anghel and S. Magdassi, ACS Nano, 2011, 5, 3354-3359.

14 D. C. Oertel, M. G. Bawendi, A. C. Arango and V. Bulović, Appl. Phys. Lett., 2005, 87, 213505.

15 J. M. Luther, M. Law, Q. Song, C. L. Perkins, M. C. Beard and A. J. Nozik, ACS Nano, 2008, 2, 271-280.

16 L. Znaidi, Mater. Sci. Eng., B, 2010, 174, 18-30.

17 K. Govender, D. S. Boyle, P. B. Kenway and P. O'Brien, J. Mater. Chem., 2004, 14, 2575-2591.

18 T. Theresa John, M. Mathew, C. Sudha Kartha, K. P. Vijayakumar, T. Abe and Y. Kashiwaba, Sol. Energy Mater. Sol. Cells, 2005, 89, 27-36.

19 S. Seki, Y. Sawada, M. Ogawa, M. Yamamoto, Y. Kagota, A. Shida and M. Ide, Surf. Coat. Technol., 2003, 169-170, 525-527.

20 U. Betz, M. Kharrazi Olsson, J. Marthy, M. F. Escolá and F. Atamny, Surf. Coat. Technol., 2006, 200, 5751-5759.

21 H. Agura, A. Suzuki, T. Matsushita, T. Aoki and M. Okuda, Thin Solid Films, 2003, 445, 263-267.

22 K. Ellmer, Nat. Photonics, 2012, 6, 809-817.

23 W. Cai, J. R. Peck, D. W. van der Weide and R. J. Hamers, Biosens. Bioelectron., 2004, 19, 1013-1019.

24 E. Galoppini, Coord. Chem. Rev., 2004, 248, 1283-1297.

25 J. Goldberger, D. J. Sirbuly, M. Law and P. Yang, J. Phys. Chem. B, 2005, 109, 9-14.

26 Y. Kajikawa, J. Appl. Phys., 2013, 114, 043719.

27 A. Tschöpe and R. Birringer, J. Electroceram., 2001, 7, 169177.

28 C. H. Ling, J. H. Fisher and J. C. Anderson, Thin Solid Films, 1972, 14, 267-288.

29 C. R. M. Grovenor, J. Phys. C: Solid State Phys., 1985, 18, 4079.

30 F. Greuter and G. Blatter, Semicond. Sci. Technol., 1990, 5, 111.

31 V. Šnejdar and J. Jerhot, Thin Solid Films, 1976, 37, 303-316.

32 A. Zabet-Khosousi and A.-A. Dhirani, Chem. Rev., 2008, 108, 4072-4124.

33 A. Celzard, J. F. Marêché, F. Payot and G. Furdin, Carbon, 2002, 40, 2801-2815.

34 D. Giaume, X. Pétrissans and P. Barboux, J. Power Sources, 2014, 272, 100-106.
35 Q. Qi, T. Zhang, Q. Yu, R. Wang, Y. Zeng, L. Liu and H. Yang, Sens. Actuators, B, 2008, 133, 638-643.

36 B. M. Kulwicki, J. Am. Ceram. Soc., 1991, 74, 697-708.

37 Z. Lu, J. Zhou, A. Wang, N. Wang and X. Yang, J. Mater. Chem., 2011, 21, 4161.

38 J. F. Chang, W. C. Lin and M. H. Hon, Appl. Surf. Sci., 2001, 183, 18-25.

39 B.-Y. Oh, M.-C. Jeong and J.-M. Myoung, Appl. Surf. Sci., 2007, 253, 7157-7161.

40 P. Lubis and M. Saito, Jpn. J. Appl. Phys., 2014, 53, 071602.

41 P. I. Djurovich, E. I. Mayo, S. R. Forrest and M. E. Thompson, Org. Electron., 2009, 10, 515-520.

42 K.-J. Euler, J. Power Sources, 1978, 3, 117-136.

43 M. Creyssels, E. Falcon and B. Castaing, Powders Grains 2009 Proc. 6th Int. Conf. Micromechanics Granul. Media, 2009, pp. 123-126.

44 J. Roncali, Chem. Rev., 1992, 92, 711-738.

45 N. Toshima and S. Hara, Prog. Polym. Sci., 1995, 20, 155-183.

46 E. E. Sheina, S. M. Khersonsky, E. G. Jones and R. D. McCullough, Chem. Mater., 2005, 17, 3317-3319.

47 K. Yao, L. Chen, Y. Chen, F. Li and P. Wang, J. Phys. Chem. C, 2012, 116, 3486-3491.

48 Y. Kim, S. Cook, S. M. Tuladhar, S. A. Choulis, J. Nelson, J. R. Durrant, D. D. C. Bradley, M. Giles, I. McCulloch, C.-S. Ha and M. Ree, Nat. Mater., 2006, 5, 197-203.

49 M. Reyes-Reyes, K. Kim and D. L. Carroll, Appl. Phys. Lett., 2005, 87, 083506.

50 T. M. Brenner, G. Chen, E. P. Meinig, D. J. Baker, D. C. Olson, R. T. Collins and T. E. Furtak, J. Mater. Chem. C, 2013, 1, 5935.

51 A. Dominguez, M. Lorke, A. L. Schoenhalz, A. L. Rosa, T. Frauenheim, A. R. Rocha and G. M. Dalpian, J. Appl. Phys., 2014, 115, 203720.

52 G. Heimel, L. Romaner, E. Zojer and J.-L. Bredas, Acc. Chem. Res., 2008, 41, 721-729.

53 H. Ishii, K. Sugiyama, E. Ito and K. Seki, Adv. Mater., 1999, 11, 605-625.

54 A. Natan, L. Kronik, H. Haick and R. T. Tung, Adv. Mater., 2007, 19, 4103-4117.

55 A. Junay, Direct synthesis of conducting polymers from simple monomers, Université Rennes 1, 2015.

56 M. T. Greiner, M. G. Helander, W.-M. Tang, Z.-B. Wang, J. Qiu and Z.-H. Lu, Nat. Mater., 2011, 11, 76-81.

57 S. Braun, W. R. Salaneck and M. Fahlman, Adv. Mater., 2009, 21, 1450-1472.

58 L. Ley, Y. Smets, C. I. Pakes and J. Ristein, Adv. Funct. Mater., 2013, 23, 794-805. 